\title{
Handover Schemes in Space Networks: Classification and Performance Comparison *
}

\author{
Pulak K Chowdhury, \\ Mohammed Atiquzzaman \\ School of Computer Science \\ University of Oklahoma \\ Norman, OK 73019-6151 \\ \{pulak, atiq\}@ou.edu
}

\author{
William Ivancic \\ Satellite Networks \& Architectures Branch \\ NASA Glenn Research Center \\ 21000 Brookpark Rd. MS 54-8 \\ Cleveland, $\mathrm{OH} 44135$ \\ wivancic@grc.nasa.gov
}

\begin{abstract}
Third Generation $(3 G)$ communication networks based on Low Earth Orbit (LEO) satellites provide a new trend in future mobile communications. LEO satellites provide lower end-to-end delays and efficient frequency spectrum utilization, making it suitable for Personal Communication Services (PCS). However, ongoing communications using LEO satellite systems experience frequent handover due to high rotational speed of satellites. In this paper, we provide a comprehensive literature survey on proposed handover schemes for LEO satellite systems. We also present a detailed classification of handover schemes in the literature. Finally, we compare the handover schemes using different Quality of Service.
\end{abstract}

\section{Introduction}

Current terrestrial wireless networks-based Personal Communication Services (PCS) provide telecommunication services within a small geographical area. To provide complete global coverage to a diverse population, several Mobile Satellite Systems (MSS), such as Iridium, Teledesic, Odyssey and ICO have been commissioned [1]. In addition to providing global coverage, MSS can connect to existing terrestrial-based telecommunication systems to share traffics overloads. The challenge with MSS is to offer quality of service similar to that provided by current terrestrial systems. Satellite systems can be classified as Geostationary Earth Orbit (GEO), Medium Earth Orbit (MEO), and Low Earth Orbit (LEO).

First generation satellites, based on GEO satellites, are deployed $35786 \mathrm{~km}$ above the equator line [1]. The position

\footnotetext{
${ }^{*}$ The research reported in this paper was funded by NASA Grant NAG3
}

and coverage area of a GEO satellite are stationary relative to a fixed location or observer on earth. Although small number (only three) of satellites at this high altitude is needed for global coverage, GEO systems exhibit some significant disadvantages for personal communication networks. A number of disadvantages, such as high power consumption at both satellites and user terminals, large propagation delay, and inefficient use of frequency spectrum, have led to the deployment of LEO satellites (Iridium, Teledesic, etc.) [1] for PCS.

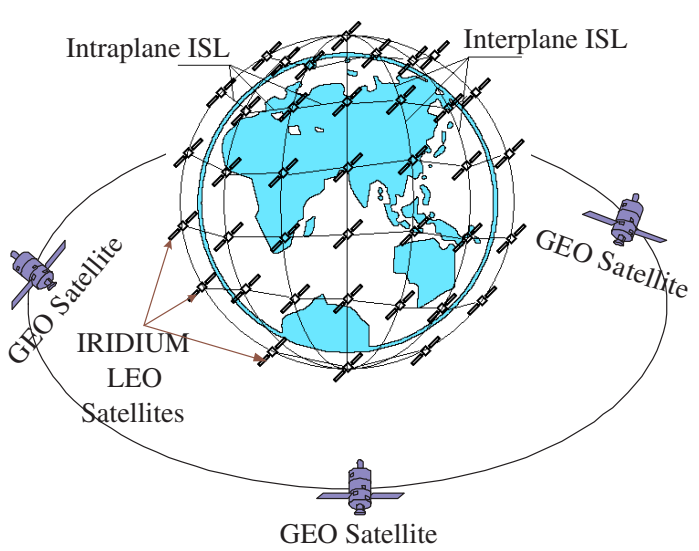

Figure 1. Mixed constellation of Iridium and GEO.

Satellites communicate among themselves using Inter Satellite Links (ISL). As shown in Fig. 1, ISLs are of two types: intraplane ISLs which connect satellites within the same orbit, and interplane ISLs connect satellites in adjacent orbits [1]. The footprint (also called cells) of a satellite is a circular area on the earth's surface [1]. To achieve efficient frequency reuse, the cells are divided into smaller cells or spotbeams (Fig. 2). The rotation of LEO satellites around the Earth result in a small visibility period of a satellite in a cell. As a result, a user terminal can be served by a number of spotbeams and satellites during the duration of a connection 
through satellites, resulting in handovers, which is significantly different from those encountered in terrestrial mobile networks. Three types of link layer handovers are observed in satellite systems [1]: (a) Spotbeam handover, (b) Satellite handover, and (c) Link handover. Spotbeam handover refers to switching between spotbeams, whereas satellite handover involves switching of a connection between satellites. Link handovers occur in the polar area due to change of connectivity patterns of satellites.

End terminals (satellites or user) which have Internet Protocol (IP) connectivity may need to change their IP address while moving, experiencing a network layer handover. When a satellite or user migrates its ongoing connections to a new IP address due to the change of coverage area of the satellite or mobility of the user, a network layer handover is required.

The objective of this paper is to provide a comprehensive survey, classification and comparison of handover schemes for the space environment. Many research have proposed different handover schemes for LEO satellite networks. Akyildiz et al. [1] categorize and describe different link layer handovers in space networks. Papapetrou et al. [13] also give a short description of different handover schemes. However, the authors are not aware of any previous work which classifies and compares both link layer and network layer handover schemes in the space environment.

The novelty of this paper is in the comparison of the relative performance and advantages of various handover schemes in the space environment; this will allow engineers to select the most appropriate handover schemes for space networks. We conclude that selection of handover schemes in space networks is dependant on the priorities of different QoS criteria for a particular scenario.

The rest of the paper is structured as follows: Sec. 2 summarizes the handover schemes in LEO satellite networks. In Sec. 3, we present the basics of spotbeam handover and classify different spotbeam handover schemes. Next, in Sec. 4, a brief introduction and classification of network layer handovers is given. Sec. 5 illustrates areas of future research in LEO satellite handover schemes. Finally, concluding remarks are presented in Sec. 6.

\section{Handover in LEO Satellite Systems}

To support continuous communication over a LEO satellite system, we may need to change one or more links as well as the IP address of the communication endpoints. Thus, both link layer and higher layer handovers may be required for satellite networking. Handovers in satellite networks can be broadly classified as:

- Link Layer Handover: Link layer handover occurs when we have to change one or more links between the communication endpoints due to dynamic connectivity patterns of LEO satellites. It can be further classified as:
- Spotbeam Handover: When the end point users cross the boundary between the neighboring spotbeams of a satellite, an intrasatellite or spotbeam handover occurs. Since the coverage area of a spotbeam is relatively small, spotbeam handovers are more frequent (every 1-2 minutes) [1].

- Satellite Handover: When the existing connection of one satellite with the end user's attachment point is transferred to another satellite, an intersatellite handover occurs.

- ISL Handover: This type of handover happens when a LEO satellite passes over the polar area. Due to the change of connectivity patterns in neighboring satellites, the inter-satellite links (ISL) have to be switched off temporarily near the polar areas. Then the ongoing connections using these ISL links have to be rerouted, causing ISL handovers.

The performance of different link layer handover schemes can be evaluated using two classic connection level QoS criteria [13]:

- call blocking probability $\left(P_{b}\right)$, the probability of a new call being blocked during handover.

- forced termination probability $\left(P_{f}\right)$, the probability of a handover call being dropped during handover.

There is a tradeoff between $P_{b}$ and $P_{f}$ in different handover schemes. The priority can be given via different treatments of new and handover calls to decrease handover call blocking [1].

- Network Layer Handover: When one of the communication endpoints (either satellite or user end) changes its IP address due to the change of coverage area of the satellite or mobility of the user terminal, a network or higher layer handover is needed to migrate the existing connections of higher level protocols (TCP, UDP , SCTP, etc.) to the new IP address. This is referred to as Network or higher layer Handover. Three different schemes can be used during this kind of handover [4]:

- Hard handover schemes: In these schemes, the current link is released before the next link is established.

- Soft handover schemes: In soft handover schemes, the current link will not be released until the next connection is established.

- Signalling Diversity schemes: Similar to soft handover. Only exception is that, in signalling diversity schemes, signalling flows through both old and new link and the user data goes through the old link during handover [4]. 
Among all the link layer handovers, spotbeam handover issues have been studied in depth in the literature, as it is the most frequent link handover experienced in LEO systems. The network layer handover has also recently received a lot of attention from the space network community. Therefore, this paper restricts itself to the classification and comparison of spotbeam handover and network layer handover schemes.

\section{Spotbeam Handover}

Dividing the footprint of an individual satellite into smaller cells or spotbeams results in better frequency utilization through the use of identical frequencies in non-adjacent spotbeams which are geographically well separated to limit interference [3]. To ensure uninterrupted ongoing communications, a current communication link should be handed off to the next spotbeam if needed. A spotbeam handover involves the release of the communication link between the user and the current spotbeam and acquiring a new link from the next spotbeam to continue the call (Fig. 2). Since both spotbeams are served by the same satellite, no other satellite is involved in the handover process.

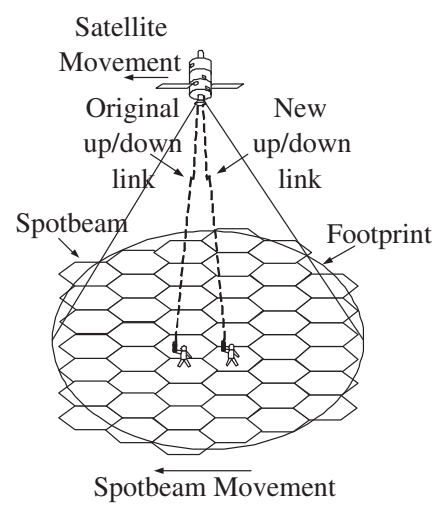

Figure 2. Spotbeam handover scenario.

Due to small spotbeams and high satellite speed, spotbeam handovers are the most common type of handovers experienced in LEO satellite systems [1]. We can consider the user mobility negligible compared to high satellite speed. As a result, the deterministic and constant movement of the satellites makes the solving of the spotbeam handover problems easier. During the handover process, if a new link or channel can not be found in the next spotbeam, the ongoing call should be dropped or blocked. From the user viewpoint, the interruption of a call is less desirable than the blocking of a newly arrived call [1]. It will be the best for a user if handovers can be guaranteed, ensuring smooth ongoing calls. Again, the selection of a suitable policy in resource management (channel allocation) can ensure new channel availability during handover. Thus, the channel allocation strategies and the handover guarantee are the prime issues in managing handover requests.

To solve spotbeam handover problem, several handover policies/schemes are proposed in the literature. We can classify the spotbeam handover schemes according to two different criteria: (a) channel allocation strategies, and (b) handover guarantee.

\subsection{Classification based on Channel Alloca- tion Strategies}

Various channel allocation strategies can be used to assign a channel to a call. Handover requests can also be considered a transferred call for the next cell, requiring allocation of a channel. Based on channel allocation strategies, handover schemes can be divided into three broad categories [16] as follows: (a) Fixed Channel Allocation (FCA) based handover schemes, (b) Dynamic Channel Allocation (DCA) based handover schemes, and (c) Adaptive Dynamic Channel Allocation (ADCA) based handover schemes. Table 1 compares different channel allocation schemes based on several link layer QoS criteria.

\subsubsection{FCA based Handover Schemes}

In FCA schemes, a set of channels is permanently assigned to each cell, according to frequency reuse distance [16]. A handover call can only be given a channel if any channel belonging to the set of the cell is available. If no channel is available, the call is blocked or, in the worst case, dropped. Fixed channel allocation schemes have a very simple implementation due to fixed predefined channel distribution [16].

An interesting variation of FCA based handover scheme is Channel Sharing Handover [8]. Channel Sharing Handover uses a channel allocation scheme called channel sharing [8], where channels can be shared between adjacent cells. A pair of adjacent cells is called a meta-cell. Two adjacent cells that form a meta-cell are called the component cells [8]. In channel sharing scheme, channels are shared between component cells to carry on the connection during handover. This scheme offers a significantly lower call blocking probability $\left(P_{b}\right)$ for the same handover dropping probability $\left(P_{f}\right)$ when compared to FCA based schemes [8].

\subsubsection{DCA based Handover Schemes}

DCA based handover schemes use dynamic channel allocation, where channels are grouped together in a central pool. Any cell requiring a channel use a channel from the pool satisfying the channel reuse distance [16]. Allocated channels are removed from the common channel pool during call time. When the call is terminated, the channel is transferred to the central pool for future reuse. DCA based schemes provide important advantage of coping up with traffic variations and overload conditions in different cells. This adaptability 
of DCA schemes makes it a fundamental channel allocation strategy in third generation cellular networks. It is concluded that there is a reduction of $P_{b}$ and $P_{f}$ in DCA compared to FCA based schemes under same conditions.

\subsubsection{ADCA based Handover Schemes}

Adaptive Dynamic Channel Allocation (ADCA) is an extension of DCA scheme (Sec. 3.1.2). It uses guard channel during handover (Handover with Guard Channel (HG), described in Sec. 3.2.2). A handover scheme with guard channel technique has to deal with the tradeoff between the number of guard channels and the number of normal channels. Excessive guard channels will create new call blocking, and fewer guard channels may block handover calls. Hence, ADCA keeps track of the current traffic load, and dynamically adapts the optimal number of guard channels according to user location information [3]. ADCA thus tries to make appropriate use of the guard channels. Cho et al. [3] proposed a new connection admission control scheme based on ADCA, called Geographical Connection Admission Control (GCAC), for LEO satellites to limit the handover blocking probability.

\subsection{Classification based on Handover Guar- antee}

A number of handover schemes provide guaranteed handover to prevent calls from being blocked or dropped during handover. Other schemes try to ensure best service by prioritizing handover over the new calls, but do not ensure any handover guarantee. Based on handover guarantee, handover schemes can be classified as: (a) Guaranteed Handover (GH) schemes, and (b) Prioritized Handover schemes.

\subsubsection{Guaranteed Handover Schemes}

In a guaranteed handover (GH) scheme, a new call is assigned a channel only if there is an available channel simultaneously in the current cell and the next transit cell. If such channels can not be found immediately, the call is blocked. As the name indicates, this scheme guarantees each handover to be successful. Maral et al. [12] proposed a guaranteed handover scheme. In that scheme, when the first handover occurs, new channel reservation request will be issued to the next candidate transit cell. If all the channels in the candidate transit cell are busy, the handover request is queued in a FIFO queue until the next handover. Thus, this scheme provides almost zero $P_{f}$ while the value of $P_{b}$ is unacceptably high. This is due to the early channel reservation (also known as channel locking in $\mathrm{GH}$ ) for a call which is still not transferred to the cell, exhibiting bad resource management. To improve resource allocation, a few modified GH schemes are proposed: (a) Elastic Handover Scheme, (b) TCRA Handover Scheme, and (c) DDBHP Scheme (compared in Table 2).

Elastic Handover Scheme: The elastic handover scheme is based on Elastic Channel Locking (ECL) scheme [17]. The idea behind the ECL scheme is that an entering call does not issue a channel locking request to the next cell immediately; instead it postpones the request for a period of time until $T_{a}$ [17]. The time $T_{a}$ is decided by the QoS requirement for handover failure probability.

TCRA based Handover Scheme: Boukhatem et al. [2] proposed a Time based Channel Reservation Algorithm (TCRA) to improve GH performance and resource utilization. TCRA locks a channel in the next candidate cell with the cell movement. TCRA is a variation of ECL (Sec. 3.2.1) except that the time instant to send the channel reservation request ( $T_{a}$ in ECL) is calculated using the estimated user location in the current cell, instead of the QoS parameters in ECL.

Dynamic Doppler Based Handover Prioritization (DDBHP) Scheme: DDBHP is yet another variation of GH scheme proposed by Papapetrou et al. [13]. This method uses Doppler effect in order to determine the terminal location, and to reserve channels at the estimated time in the next servicing cell. The system must reserve channel for the next cell in the corresponding time interval, called handover threshold $\left(t_{t H}\right)$ [13]. Clearly, different values of $t_{t H}$ will provide different level of service [13].

\subsubsection{Prioritized Handover Schemes}

Probability of handover failure is a common criteria for performance evaluation of handovers in satellite networks. In non-prioritized schemes, handover requests are treated equally as new calls, thereby increasing the probability of call dropping during handover [16]. As discussed in Sec. 3, ongoing call dropping is less desirable than new call blocking from user viewpoint. Thus, handover prioritization schemes have been proposed to decrease handover failure at the expense of increased call blocking [16]. These prioritized handover techniques can be used along with the channel allocation strategies defined in Sec. 3.1 to increase handover performance. Table 3 compares different prioritized handover schemes based on $P_{b}$ and $P_{f}$. The following are different handover prioritization categories:

Handover with Guard channel (HG): HG scheme [7] provides successful handover by reserving a set of channels (either fixed or dynamically adjustable) exclusively for 
Table 1. Comparison among channel allocation schemes

\begin{tabular}{|l|l|l|l|}
\hline Criteria & FCA & DCA & ADCA \\
\hline Complexity & $\begin{array}{l}\text { For uniform traffic condi- } \\
\text { tions, complexity is low }\end{array}$ & High & High \\
\hline$P_{b}$ & High & Low & Low \\
\hline$P_{f}$ & High & Low & Low \\
\hline $\begin{array}{l}\text { Non-uniform Traffic } \\
\text { conditions }\end{array}$ & $\begin{array}{l}\text { Complex network planning } \\
\text { required for non-uniform } \\
\text { traffic conditions }\end{array}$ & $\begin{array}{l}\text { Network planning always } \\
\text { same }\end{array}$ & $\begin{array}{l}\text { Network planning always } \\
\text { same }\end{array}$ \\
\hline $\begin{array}{l}\text { Frequency } \\
\text { reuse/Resource } \\
\text { management }\end{array}$ & No & Yes & Yes \\
\hline
\end{tabular}

Table 2. Comparison among Guaranteed Handover (GH) schemes

\begin{tabular}{|l|l|l|l|}
\hline Criteria & Elastic & TCRA & DDBHP \\
\hline Degree of guarantee & Varies with $T_{a}$ & Varies with $T_{a}$ & Varies with $T_{a}$ \\
\hline$P_{b}$ & Increases if $T_{a}$ decreases & $\begin{array}{l}\text { Depends on number of users } \\
\text { in a predefined area }\end{array}$ & Depends on $T_{a}$ \\
\hline$P_{f}$ & Decreases if $T_{a}$ increases & Null & Practically zero \\
\hline$T_{a}$ selection criteria & $\begin{array}{l}\text { QoS requirement of han- } \\
\text { dover }\end{array}$ & $\begin{array}{l}\text { Expected crossing time of } \\
\text { the user in the next cell }\end{array}$ & Doppler effect \\
\hline
\end{tabular}

handovers [16]. This reduces the probability of forced termination of calls during handover, while increasing new call blocking probability as fewer channels are available for new calls. Therefore, an important design issue is carefully choosing the number of guard channels [16].

Handover with Queueing (HQ): HQ scheme takes advantage of the overlapping area between adjacent cells [15] where a mobile host can be served by any of the cells. This makes provision of queueing the handover requests for a certain time period equal to the time of mobile host's existence in the overlapping area [16]. When a new channel becomes available, the cell checks the queue for waiting requests and grants the channel to the longest waiting request.

Several schemes, depending on the strategy to order the handover requests in the queue, have been proposed. First in first out (FIFO) scheme [15] is the most common queueing discipline where handover requests are ordered according to their arrival times. A more complex scheme called MBPS (Measurement Based Priority Scheme), is based on dynamic priority, where the handover priorities are defined by the power levels of the corresponding calls (received from the satellite) from their current spotbeam [11]. The objective is to first serve the call with the most degraded link. Another alternative priority scheme is called LUI (Last Useful Instant) scheme [15] where a handover request with a longer residual queueing time is queued ahead of other requests.
Channel Rearrangement based Handover: This scheme is only used with dynamic channel allocation schemes [15] and manages handover requests in exactly the same manner as new call attempts. Whenever a call termination occurs in a cell, the scheme performs a channel rearrangement to deallocate the channel which becomes available in the greatest number of cells.

HQ+HG Handover: HQ+HG scheme takes advantages of both guard channel and queueing schemes.

\section{Network Layer Handover}

As mentioned in Sec. 2, due to the movement of the satellites and the mobile users, the communication endpoints (user or satellites) may have to change their IP address, requiring a network layer handover. Fu et al. [6] identify two scenarios requiring network layer handover as follows:

- Satellite as a Router: In the first scenario (Fig. 3), satellites do not have any onboard equipment to produce or consume data. They merely act as routers in the Internet. Each satellite, or even a spotbeam, can be assigned an IP address. In such cases, handover between satellites (Intersatellite handover) or spotbeams (spotbeam handover) may also require network layer handover [6]. Hosts are handed over between satellites or spotbeams as they come under the footprint of a new satellite or spotbeam. 
Table 3. Comparison among prioritized handover schemes

\begin{tabular}{|l|l|l|l|l|}
\hline Criteria & HQ & HG & $\begin{array}{l}\text { Channel Rearrange- } \\
\text { ment }\end{array}$ & HQ+HG \\
\hline$P_{b}$ & $\begin{array}{l}\text { Good queueing strat- } \\
\text { egy decreases } P_{b}\end{array}$ & $\begin{array}{l}\text { Depends on guard } \\
\text { channel management }\end{array}$ & $\begin{array}{l}\text { Depends on efficient } \\
\text { channel rearrangement }\end{array}$ & $\begin{array}{l}\text { Efficient uses of HQ } \\
\text { and HG decrease } P_{b}\end{array}$ \\
\hline$P_{f}$ & $\begin{array}{l}\text { Depends on queueing } \\
\text { strategy }\end{array}$ & $\begin{array}{l}\text { Depends on guard } \\
\text { channel management }\end{array}$ & $\begin{array}{l}\text { Depends on efficient } \\
\text { channel rearrangement }\end{array}$ & $\begin{array}{l}\text { Depends on efficient } \\
\text { use of HQ and HG }\end{array}$ \\
\hline
\end{tabular}

- Satellite as a Mobile Host: In the second scenario, satellites can act as communication endpoints with all the onboard equipments which exchange data with ground stations on earth. As in Fig. 4, the satellite's footprint is moving from ground station A to B, while the satellite is bound with an IP address from ground station A. During movement, the satellite should maintain continuous connection with ground stations on earth. Thus, the IP address of the satellite has to be changed when it is handed over to ground station B, requiring network layer handover.

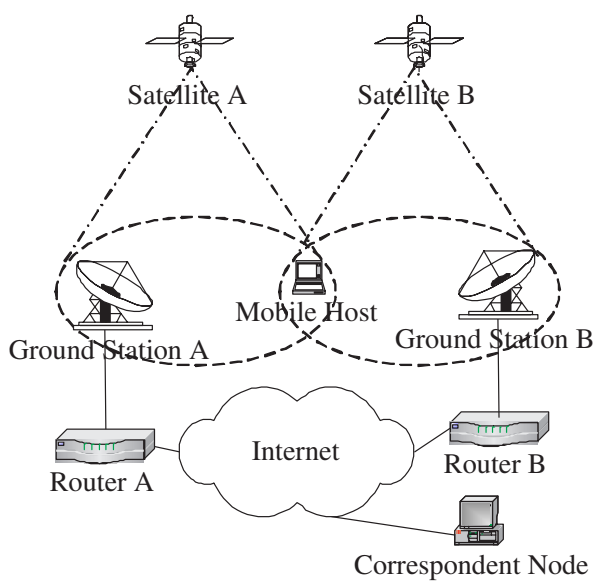

Figure 3. User handover between the satellites.

Three different strategies can be used for the network layer handovers [4]: (a) Hard handover schemes (b) Soft handover schemes (c) Signalling Diversity schemes. Table 4 compares different network layer handover schemes based on several QoS criteria.

\subsection{Hard Handover Schemes}

In hard handover schemes, the current link is released before the next link is established [4], which may result in connection blocking during handover. NASA [10] is using Mobile IP [14], which uses hard handover, to build future space communication networks.

Mobile IP (MIP) [14] is based on the concept of Home Agent (HA) and Foreign Agent (FA) (which requires modification to existing routers in Internet) for routing packets

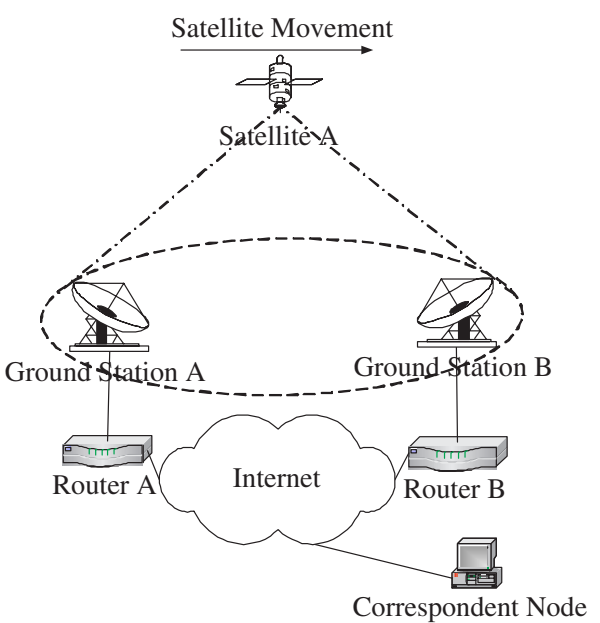

Figure 4. Satellite handover between ground stations.

from previous point of attachment to the new one. Mobile IPv6 does not need an FA as it uses IPv6 address autoconfiguration mechanism. Fig. 5 shows a Mobile IP based handover scenario where the satellite is acting as a Mobile Host (MH). When the satellite/MH determines that it is on a foreign network, it obtains a new Care of Address (CoA) from the new Foreign Agent (FA) (Ground Station B in Fig. 5). It registers the CoA address with the gateway router acting as Home Agent (HA) [5] (Fig. 5). After the registration process completes, data can be sent to the satellite using new CoA. Datagrams destined for the $\mathrm{MH}$ are intercepted by the home agent. Then, the HA tunnels the data to the FA, FA decapsulates and delivers them to the satellite. During the registration period, the $\mathrm{MH}$ is unable to send or receive packets through its previous or new point of attachment [5], giving rise to a large handover latency and high packet loss rate. Several schemes have been proposed in the literature to reduce the above mentioned drawbacks of Mobile IP based handover [14].

\subsection{Soft Handover Schemes}

During soft handover, the current connection is not released until the next connection is firmly established. Thus, both links can be used simultaneously for handover traffic management [4]. Many soft handover schemes have been proposed in the literature for terrestrial networks; for example [9] etc. The issue of adapting them into space networks 


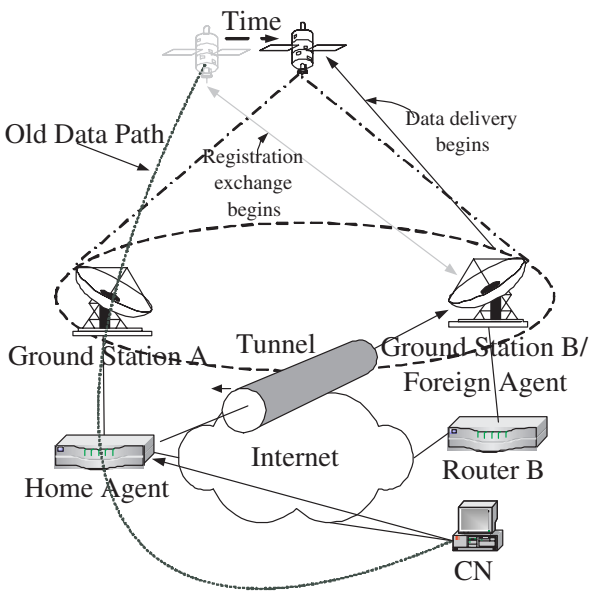

Figure 5. MIP handover.

can be investigated in future research.

\subsection{Signalling Diversity Schemes}

The signalling diversity based scheme is similar to soft handover, with the difference that the signalling procedures in signalling diversity schemes are performed through both the new and old links, while user data is sent through the old link [4]. Here no synchronization between links is needed as the old link is used for data and the new link is used for signalling.

Seamless IP diversity based Generalized Mobility Architecture (SIGMA) [6] is a signalling diversity based scheme. It is a complete transport layer mobility management scheme, and can be used with any IP diversity-based transport protocol. Fig. 6 depicts a scenario where the satellite is acting as a Mobile Host (MH). When the satellite moves into the overlapping area of two neighboring ground stations, it obtains a new IP address from the new communication agent (next visible ground station) while maintaining the old connection (via the old ground station) alive. In Fig. 6, the MH/satellite is moving from the coverage area of ground station A to ground station B. In the overlapping region, it obtains a new IP address (IP2) from ground station $\mathrm{B}$ while maintaining the connection through the old IP (IP1). The new address is used to carry all the signalling procedure to set up a new connection; during this time the mobile host can receive data via the old IP address (IP1). Whenever the received signal from ground station A drops below a certain threshold, the mobile host changes its primary address to the new one (IP2). When the mobile host leaves the overlapping area, it releases the old IP address (IP1) and continues communicating with the new address (IP2), thus achieving a smooth handover across ground stations. SIGMA reduces handover latency and data loss during handover.

Consultative Committee for Space Data Systems (CCSDS) already defined Space Communications Protocol

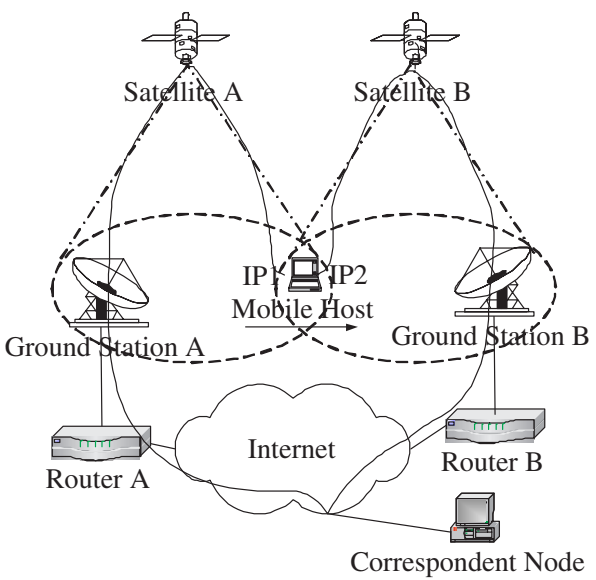

Figure 6. SIGMA handover scenario.

Specification (SCPS) for different layers of Internet protocol stack [5]. In these reports, they described the role of MIP within the proposed Next Generation Space Internet (NGSI) architecture for supporting spacecraft IP mobility [5]. SIGMA can also perform seamless network layer handover in accordance with these specifications.

\section{Future Research}

Most of the current research work on IRIDIUM [1] type constellations consider only voice traffic. But third generation satellite networks will serve all kinds of multimedia traffic including voice, video and data. QoS requirement of multimedia traffic is different from those of voice. Consequently, multimedia traffic is more difficult to serve compared to voice. As an example, video traffic is sensitive to end to end delay but can tolerate packet losses; in contrast, data traffic expects less packet losses and is insensitive to end to end delay. Consequently, handover algorithms should provide different $\mathrm{QoS}$ to serve various kinds of multimedia traffic [1]. Coupling QoS with handover management in space networks can be an active research area.

In existing handover schemes, the user mobility and earth's rotation speed are ignored. But multimedia traffic has longer call holding times than that of circuit switched traffic [1]. Handover schemes for multimedia traffic have to be designed to take into account earth's rotation speed and user's mobility in the cells.

Current research work assume that minimum number of satellites are needed for global coverage. Thus, the overlapping coverage areas of the neighboring satellites do not constitute a major portion of satellite coverage. But, in densely populated areas, for better resource management, overlapping area between the neighboring satellites can be increased [1]. This can simplify spotbeam handover management problems, since increased overlapped areas can ensure better performance of handover. Changing constellation structure in 
Table 4. Comparison among network layer handover schemes

\begin{tabular}{|l|l|l|l|}
\hline Criteria & Hard & Soft & Diversity based \\
\hline Fault Tolerant & No & Yes & Yes \\
\hline Data Loss & On the fly packets are lost & No & No \\
\hline Connection Delay & High & Low & Low \\
\hline IP Diversity & No & Yes & Yes \\
\hline
\end{tabular}

densely populated areas for better resource and handover management needs further investigation.

Compared to spotbeam handover, satellite and ISL handover issues have not been covered in details in the existing works. Developing efficient satellite and ISL handover algorithms can reduce the delay during ISL and satellite handovers.

Network layer handover issues in space networks are also recently addressed in only a few research works. Adapting current mobility management schemes for wireless networks into space networks is an area of future research. Incorporation of handover schemes from the cellular networks into space networks demands more research efforts. New efficient network layer handover schemes for space networks also need to be developed.

\section{Conclusion}

In this paper, we provide a comprehensive survey and detailed classification of handover management schemes for space networks. We conclude that selection of handover schemes in space networks is dependant on the priorities of QoS criteria for a particular scenario. SIGMA can provide network layer IP-diversity based seamless handover suitable for LEO satellite networks.

\section{References}

[1] I. F. Akyildiz, H. Uzunalioglu, and M. D. Bender. Handover management in low earth orbit (LEO) satellite networks. Mobile Networks and Applications, 4(4):301-310, December 1999.

[2] L. Boukhatem, G. Pujolle, and D. Gaiti. A time-based reservation scheme for managing handovers in satellite systems. International Journal of Network Management, 13(2):139145, March/April 2003.

[3] S. Cho, I. F. Akyildiz, M. D. Bender, and H. Uzunalioglu. A new connection admission control for spotbeam handover in LEO satellite networks. Wireless Networks, 8(4):403-415, July 2002.

[4] N. Efthymiou, Y. Hu, R. Sheriff, and A. Properzi. Inter-segment handover algorithm for an integrated terrestrial/satellite-UMTS environment. In IEEE International Symposium on Personal, Indoor and Mobile Radio Communications, PIMRC, pages 993-998, Boston, Massachusetts, USA, 8-11 September 1998.
[5] C. C. for Space Data Systems. Next generation space Internet (NGSI- supporting spacecraft IP mobility). Experimental Specification CCSDS 733.0-O-1, CCSDS Secretariat, Washington DC, USA, April 2003.

[6] S. Fu and M. Atiquzzaman. SIGMA: A transport layer mobility management scheme for terrestrial and space networks. book chapter to be published by Kluwer Academic Publishers, 2005. www.cs.ou.edu/ $\sim$ netlab.

[7] R. Guerin. Channel occupancy time distribution in a cellular radio system. IEEE Transactions on Vehicular Technology, 35(3):89-99, August 1987.

[8] S. Kalyanasundaram, E. Chong, and N. Shroff. An efficient scheme to reduce handoff dropping in LEO satellite systems. Wireless Networks, 7(1):75-85, January 2001.

[9] S. Koh, M. J. Chang, and M. Lee. mSCTP for soft handover in transport layer. IEEE Communications Letters, 8(3):189 191, March 2004.

[10] K. Leung, D. Shell, W. Ivancic, D. H. Stewart, T. L. Bell, and B. A. Kachmar. Application of mobile-IP to space and aeronautical networks. In IEEE Aerospace Conference, pages 1027-33, Big Sky, MT, USA, 10-17 March 2001.

[11] Y. Lin, S. Mohan, and A. Noerpel. Queueing priority channel assignment strategies for PCS hand-off and initial access. IEEE Transactions on Vehicular Technology, 43:704712, August 1994.

[12] G. Maral, J. Restrepo, E. D. Re, R. Fantacci, and G. Giambene. Performance analysis for a guaranteed handover service in an LEO constellation with a 'satellite-fixed cell' system. IEEE Transactions on Vehicular Technology, 47(4):1200-1214, November 1998.

[13] E. Papapetrou and F.-N. Pavlidou. QoS handover management in LEO/MEO satellite systems. Wireless Personal Communications, 24(2):189-204, February 2003.

[14] C. Perkins. Mobile networking through mobile IP. IEEE Internet Computing, 2(1):58-69, January/February 1998.

[15] E. D. Re, R. Fantacci, and G. Giambene. Handover queuing strategies with dynamic and fixed channel allocation techniques in low earth orbit mobile satellite systems. IEEE Transactions on Communications, 47(1):89-102, January 1999.

[16] V. Santos, R. Silva, M. Dinis, and J. Neves. Performance evaluation of channel assignment strategies and handover policies for satellite mobile networks. In Annual International Conference on Universal Personal Communications, pages 86-90, Tokyo, Japan, 6-10 November 1995.

[17] Y. Xu, Q. Ding, and C. Ko. Elastic handover scheme for LEO satellite mobile communication systems. In IEEE Global Telecommunications Conference, pages 1161-1165, San Francisco, CA, USA, 27 November - 1 December 2000. 